\title{
Comparison of Patient Satisfaction between Brachial Plexus Block (Axillary Approach) and General Anesthesia for Surgical Treatment of Distal Radius Fractures: A Historical Cohort Study
}

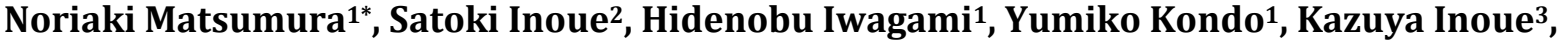 Yasuhito Tanaka ${ }^{3}$, Akihiro Okuda ${ }^{1}$}

${ }^{1}$ Department of Orthopedics, Yamato Kashihara Hospital, Kashihara, Nara, Japan

${ }^{2}$ Department of Anesthesiology, Division of Intensive Care, Nara Medical University, Kashihara, Nara, Japan

${ }^{3}$ Department of Orthopedics, Nara Medical University, Kashihara, Nara, Japan

Email: *yurayura118@gmail.com

How to cite this paper: Matsumura, N., Inoue, S., Iwagami, H., Kondo, Y., Inoue, K., Tanaka, Y. and Okuda, A. (2020) Comparison of Patient Satisfaction between Brachial Plexus Block (Axillary Approach) and General Anesthesia for Surgical Treatment of Distal Radius Fractures: A Historical Cohort Study. Open Journal of Anesthesiology, 10, 422-434.

https://doi.org/10.4236/ojanes.2020.1012037

Received: October 15, 2020

Accepted: December 7, 2020

Published: December 10, 2020

Copyright $\odot 2020$ by author(s) and Scientific Research Publishing Inc. This work is licensed under the Creative Commons Attribution International License (CC BY 4.0).

http://creativecommons.org/licenses/by/4.0/ (c) (i) Open Access

\begin{abstract}
Background: Distal radius fracture surgery is performed under general (GA) or regional anesthesia with brachial plexus block (NB). Whether anesthesia type affects patient outcomes is unclear. This study retrospectively compared patient satisfaction between GA and NB after surgery. Methods: This was a historical cohort study of 80 (34 GA and $46 \mathrm{NB}$ ) patients who underwent volar plate fixation of distal radius fractures. Propensity score analysis was used to generate a set of matched cases (NB) and controls (GA), yielding 14 matched patient-pairs. The simplified patient satisfaction scale was compared for primary outcomes. Secondary outcomes were anesthesia and surgery duration, hospital stay length, adverse events, postoperative analgesic requirement, and wrist range of motion (ROM) 2 weeks and 3 months postoperatively. Results: After propensity score matching, patients in almost all cases in both groups were "Satisfied" (effect size: $0.1, \mathrm{p}=0.572$ ), indicating little significant difference. Significant differences in adverse events and postoperative analgesic use observed before matching disappeared after matching. Anesthesia duration and hospital stay length were significantly shorter in the NB group (effect size: -1.27 and $-0.77, p=0.00074$ and $p=0.0388$, respectively), as was surgery duration (effect size: $-0.84, \mathrm{p}=0.0122$ ) after matching. Similar to before matching, wrist ROM significantly improved in the NB group (effect size: 1.11, $\mathrm{p}=0.0279$ ) in the early postoperative period, but the difference disappeared at 3 months postoperatively. Conclusions: Patient sa-
\end{abstract}


tisfaction between distal radius fracture surgery under GA and NB was similar. Nerve block could help shorten hospital stay length and surgery duration and improve postoperative functional recovery.

\section{Keywords}

Ultrasound-Guided Brachial Plexus Block (Axillary Approach), General Anesthesia, Patient Satisfaction, Distal Radius Fracture

\section{Introduction}

In Japan, the aging population is increasing, as is the number of fractures in older patients. Distal radius fractures are encountered frequently in daily clinical practice. Volar plate fixation surgery is often selected for distal radius fractures. These operations can be performed under general (GA) or local anesthesia with brachial plexus block (NB) [1] [2] [3] [4]. Egol et al. reported that NB using infraclavicular nerve block improved outcomes after distal radius fracture fixation over GA [1]. Although their results were likely to be true, they may be inconclusive because it was a retrospective analysis that was not adjusted for patient background. Wong et al. conducted a prospective randomized control trial to determine whether NB was superior to GA for surgical fixation of distal radius fractures [2]. They found that NB reduced postoperative pain after distal radial fracture fixation, with increased patient satisfaction. However, they used local wound infiltration with local anesthetics in the GA group and sedated patients with propofol in the NB group [2]. Thus, a simple comparison between NB and GA for distal radius fixation has not been performed to date. Patients who preferred GA over regional anesthesia or those scheduled to undergo regional anesthesia expressed more fear of needle puncture [5] [6]. Rundgren et al. and Galos et al. suggested that patients undergoing distal radius fracture surgery under NB complained more frequently of delayed postoperative pain [3] [4]. Therefore, it remains unclear whether the anesthesia type affects patient outcomes after volar plate fixation. Nevertheless, NB can be expected to be more beneficial than GA for several outcome measures after distal radius fracture fixation.

Patient satisfaction is a sensitive measure of a well-functioning health service system that is applicable to anesthesia services [7]. Therefore, a comparison of patient satisfaction between GA and NB can be considered a good surrogate outcome for determining which type of anesthesia is superior for volar plate fixation surgery. We conducted a clinical chart review and evaluated the role of anesthesia in patient satisfaction among surgical patients who underwent volar plate fixation surgery.

\section{Materials and Methods}

This observational cohort study was approved by the Ethics Committee of Ya- 
mato Kashihara Hospital (Kashihara, Japan; approved July 15, 2020). The need for written informed consent was waived by the institutional review board. This study was conducted as a case-control study in accordance with the recommendations of the Strengthening the Reporting of Observational studies in Epidemiology (STROBE) consensus statement [8].

\subsection{Sample Size Calculation}

Our primary outcome was patient satisfaction. Sample size calculation should have been performed for non-parametric variables. However, it would be difficult to assume a skewed distribution. It would not be unreasonable to assume that the satisfaction scale would be parametrically distributed for its sample size calculation for convenience. To calculate a post hoc sample size, a satisfaction scale difference of 1 between the type of anesthesia equal to 1 scale deviation was arbitrarily assumed. In each group, 13 patients were required to provide $90 \%$ power with a type I error probability of 0.05 . Therefore, the sample size was sufficient to detect differences in outcomes. Analyses were computed using R (version 3.0.3, R Foundation for Statistical Computing, Vienna, Austria). Statistical significance was set at $\mathrm{p}<0.05$.

\subsection{Data Handling}

Data were collected from 96 surgical cases for distal radius fractures performed at Yamato Kashihara Hospital between April 2016 and June 2020. The exclusion criteria (and reasons for consequent reductions in eligible patients) were as follows: 1) concomitant fracture surgeries $(n=9), 2)$ missing answers on the postoperative questionnaire $(\mathrm{n}=5), 3)$ age $<15$ years $(\mathrm{n}=1)$, and 4$)$ missing data sets $(\mathrm{n}=1)$ (Figure 1$)$. The remaining patient data were extracted from medical records and analyzed retrospectively.

\subsection{Perioperative Treatment}

General anesthesia was induced with intravenous propofol $\left(1-2 \mathrm{mg} \cdot \mathrm{kg}^{-1}\right)$ plus either fentanyl $\left(1-2 \mu \mathrm{g} \cdot \mathrm{kg}^{-1}\right)$ or remifentanil $\left(0.2-0.3 \mu \mathrm{g} \cdot \mathrm{kg}^{-1} \cdot \mathrm{min}^{-1}\right)$. Neuromuscular block was achieved with rocuronium $\left(0.6-0.9 \mathrm{mg} \cdot \mathrm{kg}^{-1}\right)$ for endotracheal intubation. Anesthesia was maintained with sevoflurane $(1.5 \%-2 \%)$ in a $40 \%$ oxygen and air mixture. Nitrous oxide was not used. Fentanyl (0.1 - 0.2 $\left.\mu \mathrm{g} \cdot \mathrm{kg}^{-1} \cdot \mathrm{h}^{-1}\right)$ or remifentanil $\left(0.1-0.2 \mu \mathrm{g} \cdot \mathrm{kg}^{-1} \cdot \mathrm{min}^{-1}\right)$ was used for analgesia. Following the completion of the surgical procedures, sugammadex $(2-4 \mathrm{mg} / \mathrm{kg})$ was used for the reversal of the neuromuscular blockade after status evaluation, sevoflurane was discontinued, and tracheal extubation was performed in the operating room. Regional anesthesia was performed with an ultrasound-guided brachial plexus block (axillary approach) with $0.75 \%$ ropivacaine. The median, ulnar, radial, and musculocutaneous nerves were identified with certainty, and all nerves were injected using a single puncture. The amount of ropivacaine used was 15 - $20 \mathrm{~mL}$. No other topical local anesthetics were used. A LogiQ e Premium 


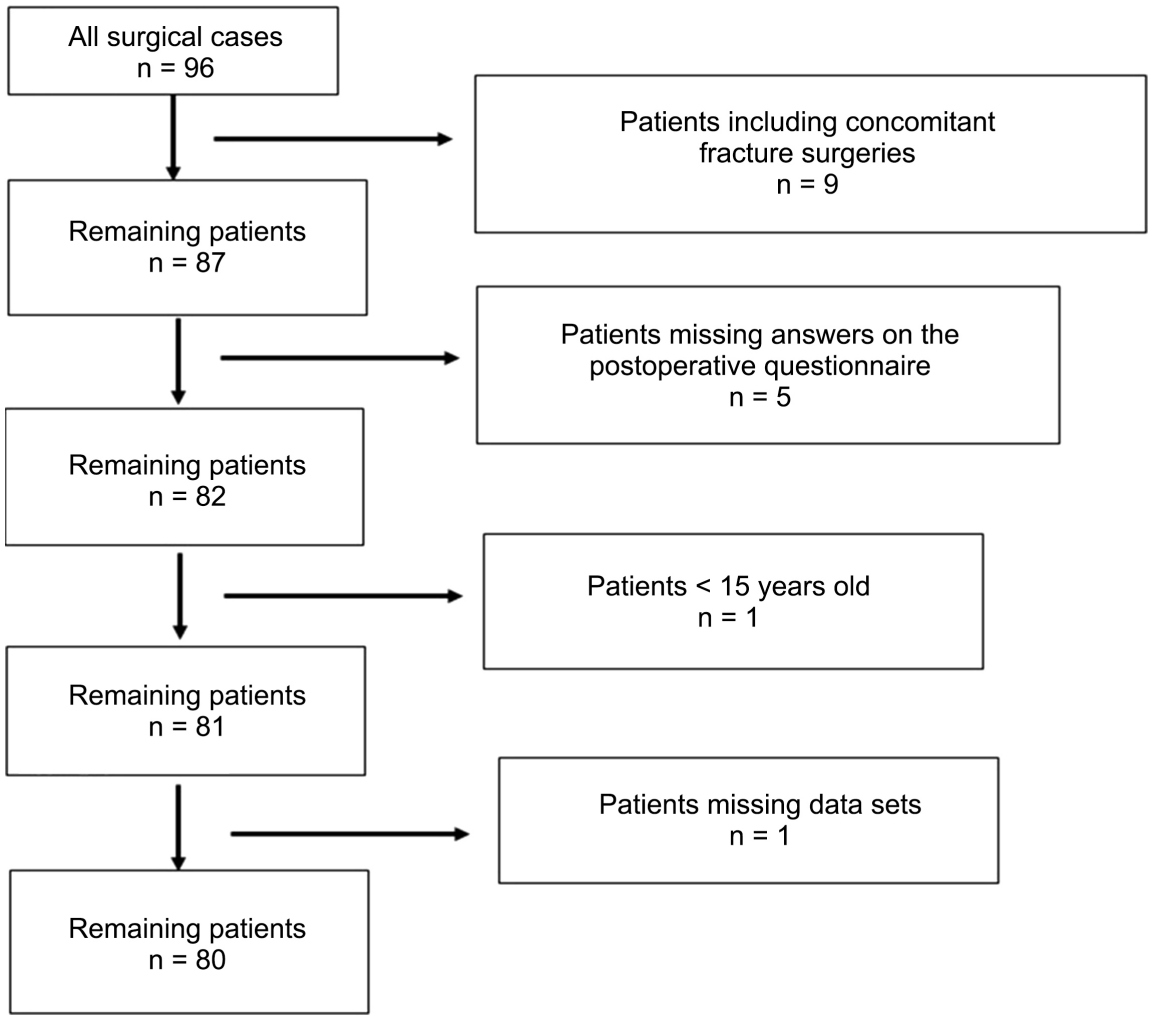

Figure 1. Flow diagram of patient enrollment.

(GE Healthcare, Tokyo, Japan) ultrasonic device with a $4-12 \mathrm{MHz}$ linear probe was used. A 23-G and 2 and 3/8-inch injection needle (Nipro, Osaka, Japan) was used.

In all cases, open surgery for fracture was performed using a volar locking plate with a volar approach of the wrist joint. All procedures were performed using a tourniquet inflated at a fixed pressure $(250 \mathrm{mmHg})$. After the surgery, splint fixation was performed for 2 days to 2 weeks.

Patients who underwent GA were transferred to the post-anesthesia care unit and stayed until the morning following surgery. Then, they were transferred to the orthopedic ward if there was no particular problem. Patients who underwent regional anesthesia were directly transferred to the orthopedic ward after surgery. The post-surgical analgesia protocol was as follows: diclofenac sodium suppositories were used when pain was severe until the following morning after surgery, and when the pain control remained poor, intramuscular injection of pentazocine was performed. Wrist range of motion (ROM) was evaluated 2 weeks and 3 months postoperatively. At discharge from the hospital, patients were asked to rate their satisfaction with the anesthesia that they received using a 5-point scale (5: Satisfied, 4: Slightly satisfied, 3: Neither agree nor disagree, 2: Slightly dissatisfied, and 1: Dissatisfied).

\subsection{Statistical Analyses}

Continuous variables are presented as mean and standard deviation (SD) if 
normally distributed or median and interquartile range (IQR) if non-normally distributed. To minimize the effect of selection bias on the outcomes and reduce distortion by confounding factors, we used propensity score matching for clinical characteristics [9] [10]. Using the propensity score analysis, we generated a set of matched cases (NB) and controls (GA). A propensity score was generated for each patient from a multivariable logistic regression model based on the covariates using data from the clinical chart as independent variables, with treatment type (GA vs. NB) as a binary dependent variable. Factors that could influence the decision of the anesthesia technique in the recorded variables were included, i.e., age, body mass index (BMI), sex, left or right arm, presence of complications (dementia, peripheral vascular disease, heart failure, chronic pulmonary disease, diabetes mellitus, chronic kidney disease, hepatic failure, and hypertension), presence of antithrombotic drugs, fracture type (classified into intra- and extraarticular fractures), and the presence of a combined ulnar fracture not requiring surgical fixation. As suggested by a review of statistical research on propensity score development, we used a structured interactive approach to refine this model to achieve covariate balance between the matched pairs [11]. Covariate balance was measured using the standardized difference, where an absolute standardized difference of 0.1 was taken as a meaningful covariate imbalance. We matched patients using a greedy matching algorithm with a caliper width of 0.01 of the estimated propensity score. A matching ratio of 1:1 was used. This procedure yielded $14 \mathrm{NB}$ patients who were propensity-matched to $14 \mathrm{GA}$ patients. For statistical inference, methods that account for the matched nature of the samples were used. For the overall incident rate, the Cochran-Mantel-Haenszel test, stratified on the matched pairs, was used to estimate the effect size (GA vs. NB). The stratified (by matched pair) paired t-test or the Wilcoxon signed-rank test was used to assess the statistical significance of the treatment effects. For the primary outcome, the simplified patient satisfaction score was compared. For the secondary outcomes, variables such as anesthesia and surgery duration, hospital stay length, adverse events (e.g., postoperative nausea and vomiting [PONV], delirium, hypoxemia, hypotension, or hypertension), the requirement of postoperative analgesics, and wrist ROM at 2 weeks and 3 months postoperatively were compared. In the unmatched population, Fisher's exact test, the Mann-Whitney $U$ test, and an unpaired t-test were used.

\section{Results}

The clinical characteristics of the 80 patients (GA and NB groups) included in this study are presented in Table 1. The side of injury, the rate of coexisting disease, the fracture type, the rate of simultaneous ulnar fracture without surgical fixation, and the rate of antithrombotic therapy were similar between the groups (absolute standardized difference: $<0.1$ ) before matching. However, variables including age, BMI, and sex were imbalanced. 
Table 1. Clinical characteristics of the two unmatched study groups.

\begin{tabular}{ccccc}
\hline & GA $(\mathrm{n}=34)$ & $\mathrm{NB}(\mathrm{n}=46)$ & ASD & p-value \\
\hline Age (median [IQR]; yr) & $63.4(18-90)$ & $67.2(31-94)$ & 0.25 & 0.271 \\
BMI (mean [SD]; kg.m $\left.{ }^{-2}\right)$ & $21.8(2.23)$ & $23.3(5.4)$ & 0.33 & 0.156 \\
Sex (female/male) & $23 / 11$ & $38 / 8$ & 0.15 & 0.183 \\
Left and right (L/R) & $20 / 14$ & $25 / 21$ & 0.02 & 0.82 \\
Fracture type (intra-/extraarticular) & $27 / 7$ & $36 / 10$ & 0 & 1 \\
Complication (no/yes) & $16 / 18$ & $22 / 24$ & 0 & 1 \\
$\begin{array}{c}\text { Ulnar fracture without requiring } \\
\text { surgical fixation (no/yes) }\end{array}$ & $20 / 14$ & $25 / 21$ & 0.02 & 0.82 \\
$\begin{array}{c}\text { Antithrombotic drug (no/yes) } \\
\text { (n) }\end{array}$ & $32 / 2$ & $42 / 4$ & 0.01 & 1 \\
\hline
\end{tabular}

GA: general anesthesia, NB: regional anesthesia with brachial plexus block, ASD: absolute standardized difference, IQR: interquartile range, BMI: body mass index, SD: standard deviation.

Patient outcomes before matching are summarized in Table 2. Patients in both groups were "Satisfied" or "Slightly satisfied" in almost all cases, and no significant difference was observed $(\mathrm{p}=0.0777)$. Adverse events, such as PONV, occurred in six patients in the GA group and in none in the NB group. Consequently, the incidence of composite adverse events was more frequently observed in the GA group ( $p=0.00448)$. There was no difference in surgery duration ( $p=0.0893$ ), but anesthesia duration and hospital stay length were significantly shorter in the NB group ( $\mathrm{p}=0.0001$ and $\mathrm{p}=0.00794$, respectively). Diclofenac sodium suppositories as postoperative analgesics were more frequently used in the NB group ( $p=0.0239)$, but the use of pentazocine was not significantly different between the groups $(\mathrm{p}=1)$. In the NB group, wrist ROM at 2 weeks postoperatively was significantly improved $(p=0.00227)$. However, there was no significant difference between the groups in wrist ROM at 3 months postoperatively $(\mathrm{p}=0.412)$.

The clinical characteristics of the two matched groups extracted by propensity score analysis are presented in Table 3. Covariates were much better balanced after matching. Most imbalanced variables in the unmatched populations were balanced after matching, except for the classification of sex.

Patient outcomes after matching are summarized in Table 4. Patients in both groups were "Satisfied" in almost all cases, and little significant difference regarding patient satisfaction between the groups was confirmed because the effect size was $0.1(\mathrm{p}=0.572)$. After matching, significant differences regarding adverse events and the use of diclofenac sodium suppositories as postoperative analgesics disappeared $(\mathrm{p}=0.1$ and $\mathrm{p}=0.343$, respectively). However, surgery duration was significantly shorter in the NB group after matching $(p=0.0122)$. Anesthesia duration and hospital stay length were also significantly shorter in 
the NB group, even after matching ( $\mathrm{p}=0.00074$ and $\mathrm{p}=0.0388$, respectively). The use of pentazocine as a postoperative analgesic did not differ between the groups $(\mathrm{p}=0.6)$. Similar to the findings before matching, wrist ROM was significantly improved in the NB group in the early postoperative period $(\mathrm{p}=0.0279)$, but the difference disappeared at 3 months after surgery $(p=0.272)$.

Table 2. Patient outcomes prior to matching.

\begin{tabular}{|c|c|c|c|c|}
\hline & $\mathrm{GA}(\mathrm{n}=34)$ & NB $(n=46)$ & Effect size & p-value \\
\hline Satisfaction score (median [IQR]) & $5(4-5)$ & $5(5-5)$ & -0.15 & 0.0777 \\
\hline Anesthesia duration (mean $[\mathrm{SD}]$; min) & $108.3(28.3)$ & $84.7(23.1)$ & 0.94 & 0.0001 \\
\hline Surgery duration (mean $[\mathrm{SD}]$; min) & $71.1(26.1)$ & $62.2(20.2)$ & 0.39 & 0.0893 \\
\hline Hospital stay length (mean [SD]; day) & $20.5(12.9)$ & $13.9(8.8)$ & 0.62 & 0.00794 \\
\hline Adverse event (no/yes) & $28 / 6$ & $46 / 0$ & 0.28 & 0.00448 \\
\hline \multicolumn{5}{|l|}{ Postoperative analgesics } \\
\hline Diclofenac sodium (no/yes) & $25 / 9$ & $22 / 24$ & 0.23 & 0.0239 \\
\hline Pentazocine (no/yes) & $31 / 3$ & $42 / 4$ & 0 & 1 \\
\hline \multicolumn{5}{|l|}{ Wrist ROM (no/yes) } \\
\hline $\begin{array}{l}2 \text { weeks postoperatively } \\
\text { (mean [SD]; degree) }\end{array}$ & $95.3(23.6)$ & $111.4(21.4)$ & 0.72 & 0.00227 \\
\hline $\begin{array}{l}3 \text { months postoperatively } \\
\text { (mean }[\mathrm{SD}] \text {; degree) }\end{array}$ & $129.3(19.3)$ & $132.7(17.6)$ & 0.19 & 0.412 \\
\hline
\end{tabular}

GA: general anesthesia, NB: regional anesthesia with brachial plexus block, IQR: interquartile range, SD: standard deviation, ROM: range of motion.

Table 3. Clinical characteristics of the two study groups after propensity score matching.

\begin{tabular}{ccccc}
\hline & GA (n=14) & NB (n=14) & ASD & p-value \\
\hline Age (median [IQR]; yr) & $64.9(20-82)$ & $64.3(39-82)$ & 0.04 & 0.867 \\
BMI (mean [SD]; kg.m $\left.{ }^{-2}\right)$ & $22.6(3.0)$ & $22.4(2.4)$ & 0.1 & 0.711 \\
Sex (female/male) & $9 / 5$ & $12 / 2$ & 0.14 & 0.45 \\
Left and right (L/R) & $7 / 7$ & $6 / 8$ & 0 & 1 \\
Fracture type (intra-/extraarticular) & $12 / 2$ & $10 / 4$ & 0.08 & 0.683 \\
Complication (no/yes) & $7 / 7$ & $8 / 6$ & 0 & 1 \\
Ulnar fracture without requiring & & & 0 & 1 \\
surgical fixation (no/yes) & $7 / 7$ & $7 / 7$ & 0 & 1 \\
Antithrombotic drug (no/yes) & $13 / 1$ & $13 / 1$ & 0 & \\
\hline
\end{tabular}

GA: general anesthesia, NB: regional anesthesia with brachial plexus block, ASD: absolute standardized difference, IQR: interquartile range, BMI: body mass index, SD: standard deviation. 
Table 4. Patient outcomes after propensity matching.

\begin{tabular}{|c|c|c|c|c|}
\hline & $\mathrm{GA}(\mathrm{n}=14)$ & NB $(n=14)$ & Effect size & p-value \\
\hline Satisfaction score (median [IQR]) & $5(5-5)$ & $5(5-5)$ & 0.1 & 0.572 \\
\hline Anesthesia duration (mean [SD]; min) & $110.4(29.2)$ & $73.4(14.3)$ & -1.27 & 0.00074 \\
\hline Surgery duration (mean $[\mathrm{SD}] ;$ min) & $73.2(24.5)$ & $52.6(13.2)$ & -0.84 & 0.0122 \\
\hline Hospital stay length (mean [SD]; day) & $21.1(13.3)$ & $10.9(7.8)$ & -0.77 & 0.0388 \\
\hline Adverse event (no/yes) & $12 / 2$ & $14 / 0$ & 0.19 & 0.1 \\
\hline \multicolumn{5}{|l|}{ Postoperative analgesics } \\
\hline Diclofenac sodium (no/yes) & $10 / 4$ & $6 / 8$ & 0.18 & 0.343 \\
\hline Pentazocine (no/yes) & $12 / 2$ & $14 / 0$ & 0.11 & 0.6 \\
\hline \multicolumn{5}{|l|}{ Wrist ROM (no/yes) } \\
\hline $\begin{array}{l}2 \text { weeks postoperatively } \\
\text { (mean }[\mathrm{SD}] ; \text { degree) }\end{array}$ & $94.3(22.8)$ & $119.6(23.4)$ & 1.11 & 0.0279 \\
\hline $\begin{array}{l}3 \text { months postoperatively } \\
\text { (mean [SD]; degree) }\end{array}$ & $127.9(21.7)$ & $135.4(15.9)$ & 0.35 & 0.272 \\
\hline
\end{tabular}

GA: general anesthesia, NB: regional anesthesia with brachial plexus block, IQR: interquartile range, SD: standard deviation, ROM: range of motion.

\section{Discussion}

Surgery for distal radius fractures using the volar plate is common, and in recent years, ultrasound-guided nerve block anesthesia has been widely used for this surgery. We found that outcome measures such as surgery duration, hospital stay length, and wrist ROM at 2 weeks postoperatively were superior in the NB group than in the GA group. Postoperative analgesic use was more frequently observed in the NB group before matching; however, the difference disappeared after matching. It is reasonable to conclude that propensity score matching was appropriately performed because imbalanced variables, including BMI and age, which have been suggested to be related to difficult regional nerve block [12] [13], were adjusted after matching.

Gamo et al. reported that $97.6 \%$ of patients undergoing orthopedic surgery on the upper limbs with an ultrasound-guided supraclavicular brachial plexus block were satisfied with the anesthesia that they received [14]. However, few studies have compared patient satisfaction with GA with respect to the type of anesthesia they received. Wong et al. confirmed that NB increased patient satisfaction. However, they did not simply or directly compare NB with GA [2]. Therefore, we conducted this study on patient satisfaction based on the hypothesis that NB would be superior to GA. However, we failed to find the superiority of NB over GA with regard to patient satisfaction with the anesthesia administration. Both groups were highly satisfied with the anesthesia that they received. In our hospital, we give patients the option to choose the type of anesthesia after a detailed 
explanation of the treatment options. Therefore, patients may have been satisfied with their decisions. This is reasonable and convincing because shared decision-making has been reported to improve healthcare outcomes even in the field of surgery [15].

Interestingly, surgery duration was significantly shorter in the NB group than in the GA group. Previous studies showed no difference in surgery duration between the NB and GA groups [2] [3] [4]. The surgical procedures were the same; therefore, the cause of the shortened surgery duration observed in our study is unknown. In the NB group, patients were awake, and surgical staff refrained from non-surgery-related conversation which was reported to be associated with a significantly higher sound level and consequent lack of concentration [16]. Therefore, the absence of noisy non-surgery-related conversation might have facilitated the surgical procedures, which should be beneficial for patients. Otherwise, this may have been the result of the surgeon's desire to end the operation as soon as possible, considering the tourniquet time during the awake status. Anesthesia duration was also significantly shorter in the NB group than in the GA group. This result may be due to the obscure definition of anesthesia duration in cases of regional nerve block. All patients who underwent NB were in the orthopedic ward prior to transfer to the operating room. In such cases, the time required to perform regional anesthesia was not included. However, the time used for NB was usually 5 - $10 \mathrm{~min}$, which included the time for aseptic preparations. The time required for NB was thought to be much shorter than the time for the induction of and recovery from GA.

Hospital stay length was significantly shorter in the NB group than in the GA group. This study was conducted in Japan, where the length of hospitalization is relatively long [17]. The Japanese universal public insurance system is relatively generous in its support of hospitalization. Therefore, hospitalization in Japan is usually determined not only by the patient's medical status but also by the patient's social background. Therefore, the results cannot be generalized for countries with generally shorter hospitalizations. However, shorter hospitalization certainly contributes to the reduction of medical care costs even if the Japanese universal public insurance system is applied. Additionally, considering that GA costs approximately $\$ 600$ (US) in Japan while regional nerve block costs $\$ 15$, it is thought that regional nerve block is superior in terms of value for money.

The incidence rate of composite adverse events did not differ between the groups. PONV is observed more frequently after GA [2] [4]. This is a reasonable phenomenon because the consensus guidelines for PONV recommend avoiding GA to prevent PONV [18]. In our study, some patients in the GA group suffered from PONV; however, we did not reach a statistical conclusion because of the small sample size.

Conversion from NB to GA is sometimes required in cases of insufficient effect of the nerve block [2] [4]. However, no patients required conversion to GA in our study cohort. Previous studies have used supra- or infra-clavicular nerve 
block, which allows simple injection of local anesthetics into the sheath containing the nerves [19]. However, the infiltration of local anesthetics into each nerve in the sheath might vary. On the other hand, the axillary approach for brachial plexus block requires the injection of agents around each of the four nerves, which might allow agents to infiltrate more homogenously [20]. This might be the reason that no patients converted to GA in our study cohort.

In the raw data analysis, the NB group used diclofenac more frequently during the postoperative period than the GA group. There was still a trend toward this phenomenon in the matched data analysis; however, a statistically significant difference was not observed after matching. This is inconclusive because the statistical power was not sufficient, and this study was not primarily conducted to answer this question. Nevertheless, this concern has been discussed in the field of regional anesthesia as so-called "rebound pain," which can occur after NB effectiveness wears off [2] [3] [4]. The timing of postoperative diclofenac use was 8 - 12 hours after the injection of local anesthetics. Rundgren et al. suggested that day-surgery patients who receive NB should be informed about the intense rebound pain that may occur after discharge [4]. Galos et al. also recommended that patients be made aware of the rebound pain phenomenon after regional anesthesia [3]. Additionally, Wong et al. suggested that regular preventive analgesic medications, even in the absence of pain and patient education, can attenuate rebound pain [2]. Therefore, preventive analgesics could have treated rebound pain more appropriately in our cohort.

Long-term postoperative functional outcomes have been reported to be similar between NB and GA [3] [4]. The findings in our cohort were the same. However, the short-term functional outcomes were better in the NB group than in the GA group. Additionally, functional outcomes in the NB group improved more favorably regardless of the fracture type. The reason for this phenomenon remains unknown because the analgesic effects of NB should have surely ceased on starting the postoperative rehabilitation program. Generally, it is expected that regional anesthesia can modulate the inflammatory response mainly through the direct anti-inflammatory effects of local anesthesia and the effective block of neural afferents and sympathetic activation [21]. An animal study reported that a single dose of nerve block attenuated inflammation in the early postoperative period after surgery in the humeral fracture fixation model compared to GA [22]. This suggests that nerve block might have some favorable effects on functional outcomes, which cannot be explained by analgesic effects only, in the early postoperative period.

This study had several limitations that should be addressed. To minimize the effect of selection bias on the outcomes, propensity score matching for clinical characteristics was used to reduce distortion by the confounding factors. Nevertheless, in this retrospective study, unmeasured variables could still have confounded the results. Therefore, several variables may have affected the primary outcome. Furthermore, in propensity matching, not all absolute differences for 
covariates reached a value of $<0.1$. The imbalance of the cohort because of the limited number of patients cannot be ruled out and could have affected the analysis. However, the p-value was 0.45 , and the very small effect size (0.14) for the classification of sex may be clinically negligible. A larger cohort study reported a greater proportion of women in the block group than in the general group [1]. Women may prefer regional anesthesia to GA. Finally, a counterargument that a larger population might have resulted in different outcomes may be proposed. This objection might be true; however, it could be difficult to observe a different primary outcome simply by increasing the sample size because both groups were maximally satisfied with the anesthesia program.

\section{Conclusion}

In conclusion, anesthesia for distal radius fracture fixation with ultrasound-guided brachial plexus block (axillary approach) was equivalent to GA in terms of patient satisfaction in our cohort. Nerve block shortened the hospital stay length and surgery duration. Even a single dose of nerve block might work favorably for early postoperative functional outcomes. Nevertheless, we may need to be cognizant of so-called "rebound pain" in case of a single dose of nerve block.

\section{Acknowledgements}

We would like to thank Editage (http://www.editage.com/) for English language editing.

\section{Conflicts of Interest}

The authors declare no conflicts of interest regarding the publication of this paper.

\section{References}

[1] Egol, K.A., Soojian, M.G., Walsh, M., Katz, J., Rosenberg, A.D. and Paksima, N. (2012) Regional Anesthesia Improves Outcome after Distal Radius Fracture Fixation over General Anesthesia. Journal of Orthopaedic Trauma, 26, 545-549. https://doi.org/10.1097/BOT.0b013e318238becb

[2] Wong, S.S., Chan, W.S., Fang, C., Chan, C.W., Lau, T.W., Leung, F. and Cheung, C.W. (2020) Infraclavicular Nerve Block Reduces Postoperative Pain after Distal Radial Fracture Fixation: A Randomized Controlled Trial. BMC Anesthesiology, 20, Article No. 130. https://doi.org/10.1186/s12871-020-01044-4

[3] Galos, D.K., Taormina, D.P., Crespo, A., Ding, D.Y., Sapienza, A., Jain, S. and Tejwani, N.C. (2016) Does Brachial Plexus Blockade Result in Improved Pain Scores After Distal Radius Fracture Fixation? A Randomized Trial. Clinical Orthopaedics and Related Research, 474, 1247-1254. https://doi.org/10.1007/s11999-016-4735-1

[4] Rundgren, J., Mellstrand Navarro, C., Ponzer, S., Regberg, A., Serenius, S. and Enocson A. (2019) Regional or General Anesthesia in the Surgical Treatment of Distal Radial Fractures: A Randomized Clinical Trial. The Journal of Bone and Joint Surgery, 101, 1168-1176. https://doi.org/10.2106/JBJS.18.00984 
[5] Lavado, J.S., Gonçalves, D., Gonçalves, L., Sendino, C. and Valente, E. (2019) General or Regional? Exploring Patients' Anaesthetic Preferences and Perception of Regional Anaesthesia. Revista Española de Anestesiología y Reanimación, 66, 199-205. https://doi.org/10.1016/j.redar.2018.12.004

[6] Bheemanna, N.K., Channaiah, S.R.D., Gowda, P.K.V., Shanmugham, V.H. and Chanappa, N.M. (2017) Fears and Perceptions Associated with Regional Anesthesia: A Study from a Tertiary Care Hospital in South India. Anesthesia Essays and Researches, 11, 483-488. https://doi.org/10.4103/aer.AER 5117

[7] Heidegger, T., Saal, D. and Nübling, M. (2013) Patient Satisfaction with Anaesthesia -Part 1: Satisfaction as Part of Outcome- and What Satisfies Patients. Anaesthesia, 68, 1165-1172. https://doi.org/10.1111/anae.12347

[8] Vandenbroucke, J.P., von Elm, E. and Altman, D.G. (2007) Strengthening the Reporting of Observational Studies in Epidemiology (STROBE): Explanation and Elaboration. Academia and Clinic, 147, W163-W194. ttps://doi.org/10.7326/0003-4819-147-8-200710160-00010-w1

[9] D’Agostino Jr., R.B. (1998) Propensity Score Methods for Bias Reduction in the Comparison of a Treatment to a Non-Randomized Control Group. Statistics in Medicine, 17, 2265-2281.

https://doi.org/10.1002/(SICI)1097-0258(19981015)17:19<2265::AID-SIM918>3.0.C $\underline{\mathrm{O} ; 2-\mathrm{B}}$

[10] Rubin, D.B. (1997) Estimating Causal Effects from Large Data Sets Using Propensity Scores. Annals of Internal Medicine, 127, 757-763. https://doi.org/10.7326/0003-4819-127-8 Part 2-199710151-00064

[11] Austin, P.C. (2007) Propensity-Score Matching in the Cardiovascular Surgery Literature from 2004 to 2006: A Systematic Review and Suggestions for Improvement. The Journal of Thoracic and Cardiovascular Surgery, 134, 1128-1135. https://doi.org/10.1016/j.jtcvs.2007.07.021

[12] Schroeder, K., Andrei, A.C., Furlong, M.J., Donnelly, M.J., Han, S. and Becker, A.M. (2012) The Perioperative Effect of Increased Body Mass Index on Peripheral Nerve Blockade: An Analysis of 528 Ultrasound Guided Interscalene Blocks. Brazilian Journal of Anesthesiology, 62, 28-38. https://doi.org/10.1016/S0034-7094(12)70100-9

[13] Ružman, T., Gulam, D., Haršanji Drenjančević, I., Venžera-Azenić, D., Ružman, N. and Burazin, J. (2014) Factors Associated with Difficult Neuraxial Blockade. Local and Regional Anesthesia, 7, 47-52. https://doi.org/10.2147/LRA.S68451

[14] Gamo, K., Kuriyama, K., Higuchi H., Uesugi, A., Nakase, T., Hamada, M. and Kawai, H. (2014) Ultrasound-Guided Supraclavicular Brachial Plexus Block in Upper Limb Surgery: Outcomes and Patient Satisfaction. The Bone \& Joint Journal, 96B, 795-799. https://doi.org/10.1302/0301-620X.96B6.31893

[15] Niburski, K., Guadagno, E., Abbasgholizadeh-Rahimi, S. and Poenaru, D. (2020) Shared Decision Making in Surgery: A Meta-Analysis of Existing Literature. Patient, 13, 667-681. https://doi.org/10.1007/s40271-020-00443-6

[16] Kurmann, A., Peter, M., Tschan, F., Mühlemann, K., Candinas, D. and Beldi, G. (2011) Adverse Effect of Noise in the Operating Theatre on Surgical-Site Infection. British Journal of Surgery, 98, 1021-1025. https://doi.org/10.1002/bjs.7496

[17] Organisation for Economic Co-Operation and Development (2019) Length of Hospital Stay (Indicator). https://data.oecd.org/healthcare/length-of-hospital-stay.htm.

[18] Gan, T.J., Belani, K.G., Bergese, S., Chung, F., Diemunsch, P., Habib, A.S., Jin, Z., Kovac, A.L., Meyer, T.A., Urman, R.D., Apfel, C.C., Ayad, S., Beagley, L., Candiotti, 
K., Englesakis, M., Hedrick, T.L., Kranke, P., Lee, S., Lipman, D., Minkowitz, H.S., Morton, J. and Philip, B.K. (2020) Fourth Consensus Guidelines for the Management of Postoperative Nausea and Vomiting. Anesthesia \& Analgesia, 131, 411-448. https://doi.org/10.1213/ANE.0000000000004833

[19] Vander Beek, J. (2009) Supraclavicular Brachial Plexus Block. In: Vander Beek, J., Ed., The Neuraxiom Playbook of 9 Essential Blocks. A Handbook of Ultrasound Guided Regional Nerve Blocks, 1st Edition, Neuraxiom LLC, Olympia, 74-80.

[20] Vander Beek, J. (2009) Axillary Brachial Plexus Block. In: Vander Beek, J., Ed., The Neuraxiom Playbook of 9 Essential Blocks: A Handbook of Ultrasound Guided Regional Nerve Blocks, 1st Edition, Neuraxiom LLC, Olympia, 86-91.

[21] Bugada, D., Ghisi, D. and Mariano, E.R. (2017) Continuous Regional Anesthesia: A Review of Perioperative Outcome Benefits. Minerva Anestesiologica, 83, 1089-1100.

[22] He, Y., Li, Z. and Zuo, Y.-X. (2015) Nerve Blockage Attenuates Postoperative Inflammation in Hippocampus of Young Rat Model with Surgical Trauma. Mediators of Inflammation, 2015, Article ID: 460125. https://doi.org/10.1155/2015/460125 